\title{
Are quality of life outcomes comparable following stereotactic radiotherapy and minimally invasive surgery for stage I lung cancer patients?
}

\author{
Cecilia Pompili ${ }^{1}$, Kate Absolom ${ }^{1}$, Kevin Franks $^{2}$, Galina Velikova $^{1}$ \\ ${ }^{1}$ Section of Patient Centred Outcomes Research, Leeds Institute of Cancer and Pathology, University of Leeds, Leeds, UK; ${ }^{2}$ Clinical Oncology, St \\ James' University Hospital, Leeds, UK \\ Contributions: (I) Conception and design: All authors; (II) Administrative support: None; (III) Provision of study materials or patients: None; (IV) \\ Collection and assembly of data: C Pompili; (V) Data analysis and interpretation: All authors; (VI) Manuscript writing: All authors; (VII) Final \\ approval of manuscript: All authors. \\ Correspondence to: Cecilia Pompili. Section of Patient Centred Outcomes Research, University of Leeds, St James' Institute of Oncology, Beckett \\ Street, Leeds LS9 7TF, UK. Email: c.pompili@leeds.ac.uk.
}

\begin{abstract}
A best evidence topic in thoracic surgery was produced in accordance with published guidelines. The question addresses the effect of stereotactic ablative radiotherapy (SABR) and minimally-invasive anatomical lung resection on quality of life $(\mathrm{QoL})$ in patients with stage I non-small-cell lung cancer. Altogether more than 428 papers were found using the reported search. Only one small RCT of 22 patients was identified that addressed the effect of SABR and surgery on QoL and found global health status to be statistically significantly worse for surgical patients when compared to SABR. Sixteen further studies provided some supporting evidence, but not directly compared QoL between the two treatment modalities. Consequently, there is no general consensus currently available from the literature. Among the nine SABR-only studies, only five specified the percentage of patients who were medically operable but refused surgery. None of the studies identified significant difference in most of the QoL domains 12 months after treatment. Within the surgical papers, patients recovered well after video-assisted thoracoscopic surgical (VATS) anatomical lung resection for stage-I NSCLC. Confirming previous evidence of open surgery, three studies demonstrated worsening of QoL domains from 8 weeks to 3 months and a return to baseline after 12 months. Emotional functioning (EF) showed an improvement across both treatments, often superseding baseline scores. Given the different population characteristics of all the available evidence, further appropriately powered and randomised studies are necessary to clarify this issue.
\end{abstract}

Keywords: Patient Reported Outcomes Measures (PROMS); quality of life (QoL); lung cancer; stereotactic ablative radiotherapy (SABR); video-assisted thoracoscopic surgical (VATS); BET

Submitted Sep 03, 2018. Accepted for publication Nov 15, 2018.

doi: $10.21037 /$ jtd.2018.11.89

View this article at: http://dx.doi.org/10.21037/jtd.2018.11.89

\section{Introduction}

A best evidence topic was conducted according to recognised guidelines in the surgical field (1).

\section{Clinical scenario}

An 80-year-old male patient presents to your clinic complaining of shortness of breath and weight loss. Preoperative staging confirms a peripheral stage-I NSCLC in the right upper lobe. The lung function test returns a forced expiratory volume in 1 second (FEV1) of 50\% and diffusing capacity of $40 \%$. The cardio-pulmonary exercise test (CPEX) showed a VO2Max of $11.5 \mathrm{~mL} / \mathrm{kg} / \mathrm{min}$.

Although a lobectomy would represent the standard 
oncological treatment, tests indicated high surgical risk. You discuss with the patient possible alternative non-surgical treatment, including stereotactic ablative radiotherapy (SABR). The patient asks how these two treatments will affect his quality of life (QoL). You resolve to check the literature yourself.

\section{Three-part question}

In (patients with stage I non-small-cell lung cancer) what effect do (SABR and minimally-invasive surgery) have on $(\mathrm{QoL})$ ?

\section{Search strategy and study selection}

The English language scientific literature was reviewed primarily by searching MEDLINE and EMBASE from 1996 through December 2017 using the Ovid Interface: [quality of life.mp OR patient reported outcome.mp OR eortc qlq.mp OR short-form 36.mp] AND [surger*.mp OR lobectom*.mp. OR segmentectomy.mp OR sleeve resection. mp] AND [Stereotactic ablative radiotherapy.mp. OR SABR.mp OR sbrt.mp] AND [NSCLC.mp OR lung cancer. mp OR stage I]. Studies with less than $20 \%$ video-assisted thoracoscopic surgical (VATS) procedures were excluded.

Four hundred and twenty-eight papers were found using the reported search. From these, only one small randomized controlled trial (RCT) was identified that provided evidence addressing the specific question: in patients with stage I nonsmall-cell lung cancer what effect do SABR and minimallyinvasive surgery have on QoL? Sixteen studies provided supporting evidence, as not directly comparing QoL in these two treatment modalities. All the seventeen studies appraised in this manuscript are summarised in Table 1.

\section{Results}

Sixteen studies were identified, which separately investigated the effect of SABR or VATS lobectomy for early stage NSCLC on QoL. Only one RCT of 22 patients has been identified which directly compared the QoL outcomes of medically operable stage IA NSCLC patients treated with either SABR or surgery (2). Time to deterioration (TTD) in QoL domains was used to detect changes during the followup. It was calculated from the time of randomization to first appearance of a clinical significant difference in QoL scores. Validated instruments at baseline, and up to 24 months post-treatment were used: the European Organization for
Research and Treatment of Cancer QoL Core questionnaire (EORTC QLQ-C30) and its lung cancer supplement (LC-13).

Patients without a documented clinically meaningful difference in Patient Reported Outcomes Measures (PROMS) were censored at the time of last PROM assessment.

The authors found similar results in most of the QoL scales. They concluded that SABR may have advantages in the global QoL and indirect cost of productivity loss. However, only TTD of global health status was found to be significantly worse on univariable COX proportional hazard modelling for surgical patients when compared to SABR.

\section{SABR studies}

Out of nine evaluating the impact of SABR on QoL, only five studies specified the percentage of patients who refused surgery. In all the other studies patients who had the SABR treatment were patients considered medically inoperable and therefore generally with worse comorbidities and poorer cardio-pulmonary functions than patients undergoing to surgery.

Lagerwaard et al. (3) conducted the largest study on 382 patients over a period of 24 months. Physical functioning was the only QoL domain to statistically significantly worsen, though by less than the clinical meaningful significance of 10 points (19). Physical functioning in fact decreased by more than 10 points in $26 \%$ of patients, remained stable in $53 \%$, and had improved in $22 \%$ after 1 year.

Mathieu (4) reported favourable long-term QoL and pulmonary function in 45 patients treated with SABR with a follow-up longer than 3 years. They also reported a QLQ-LC30 emotional score improvement at 36 months. However, the exclusion of patients with recurrent disease may have affected the QoL results.

Ubels et al. (5) prospectively studied QoL in 39 inoperable patients for 5 years. Although the emotional functioning (EF) scores improved significantly, dyspnea slowly worsened 2 years after SABR. The trajectory of the global health showed that it was near the baseline value during the first year, improved at 18 months and then significantly declined to the baseline value during the next years.

One of the first studies to explore the QoL after SABR treatment was from van der Voort van Zyp et al. (6). The only significant change observed was an improvement in EF. 


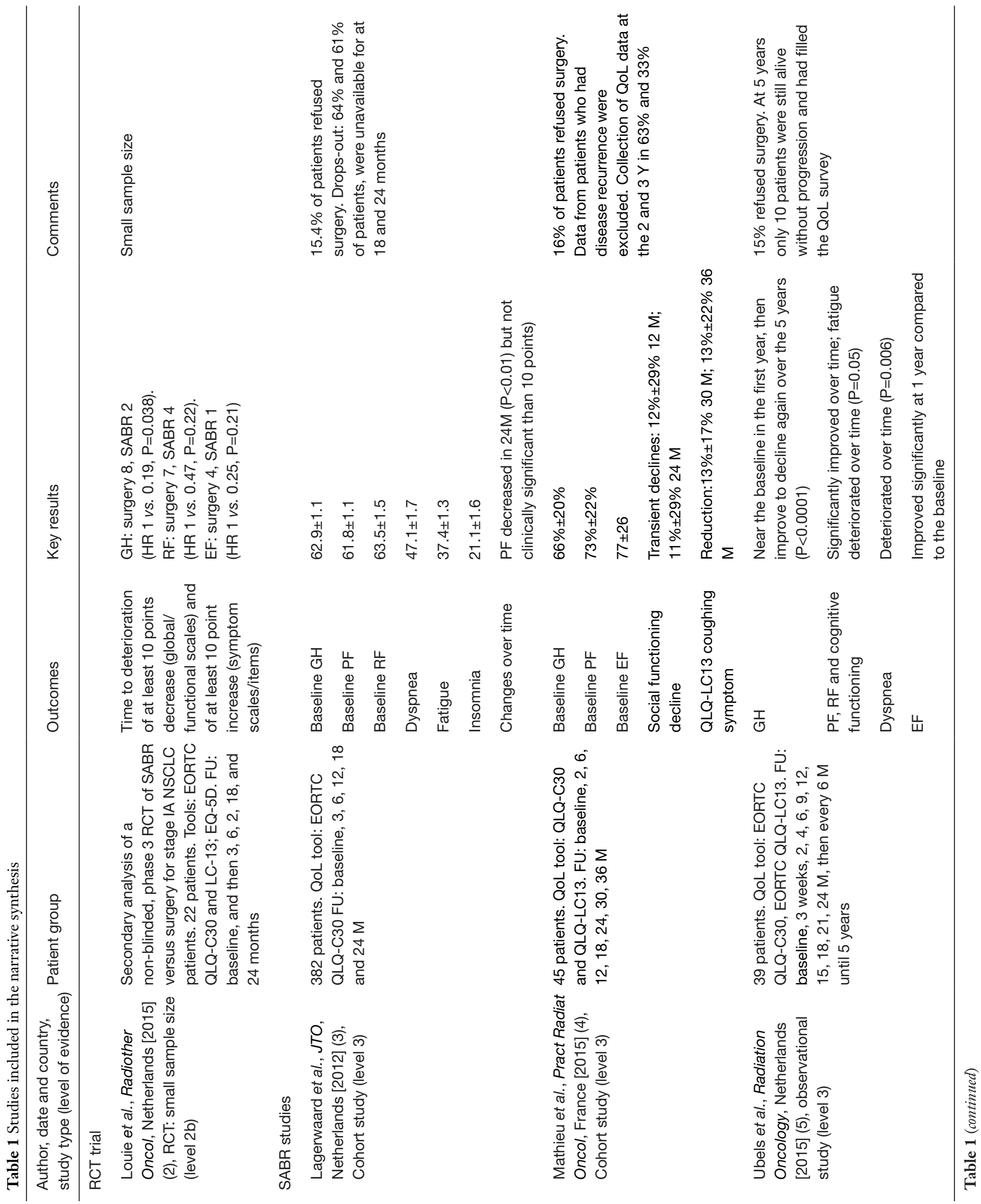




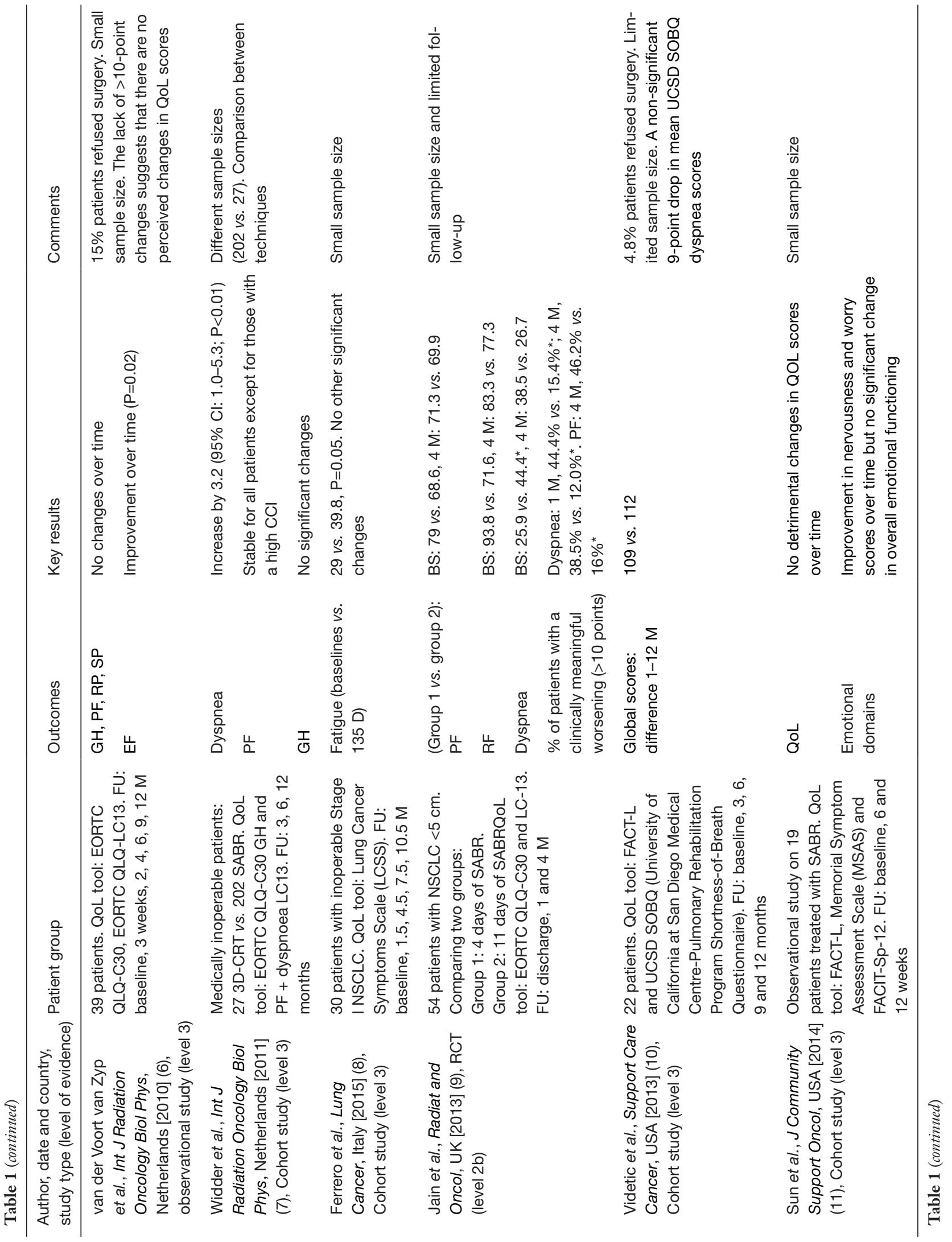




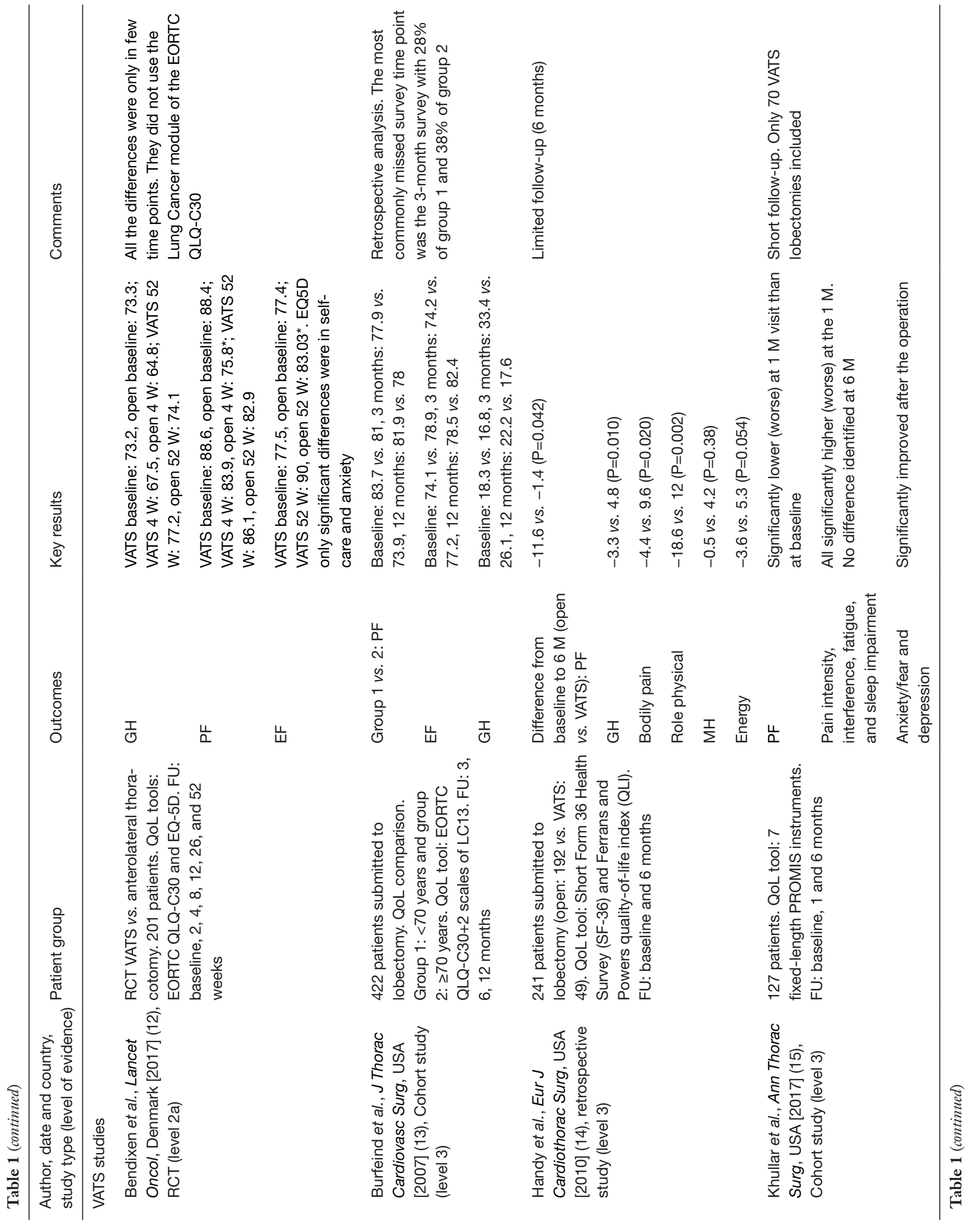




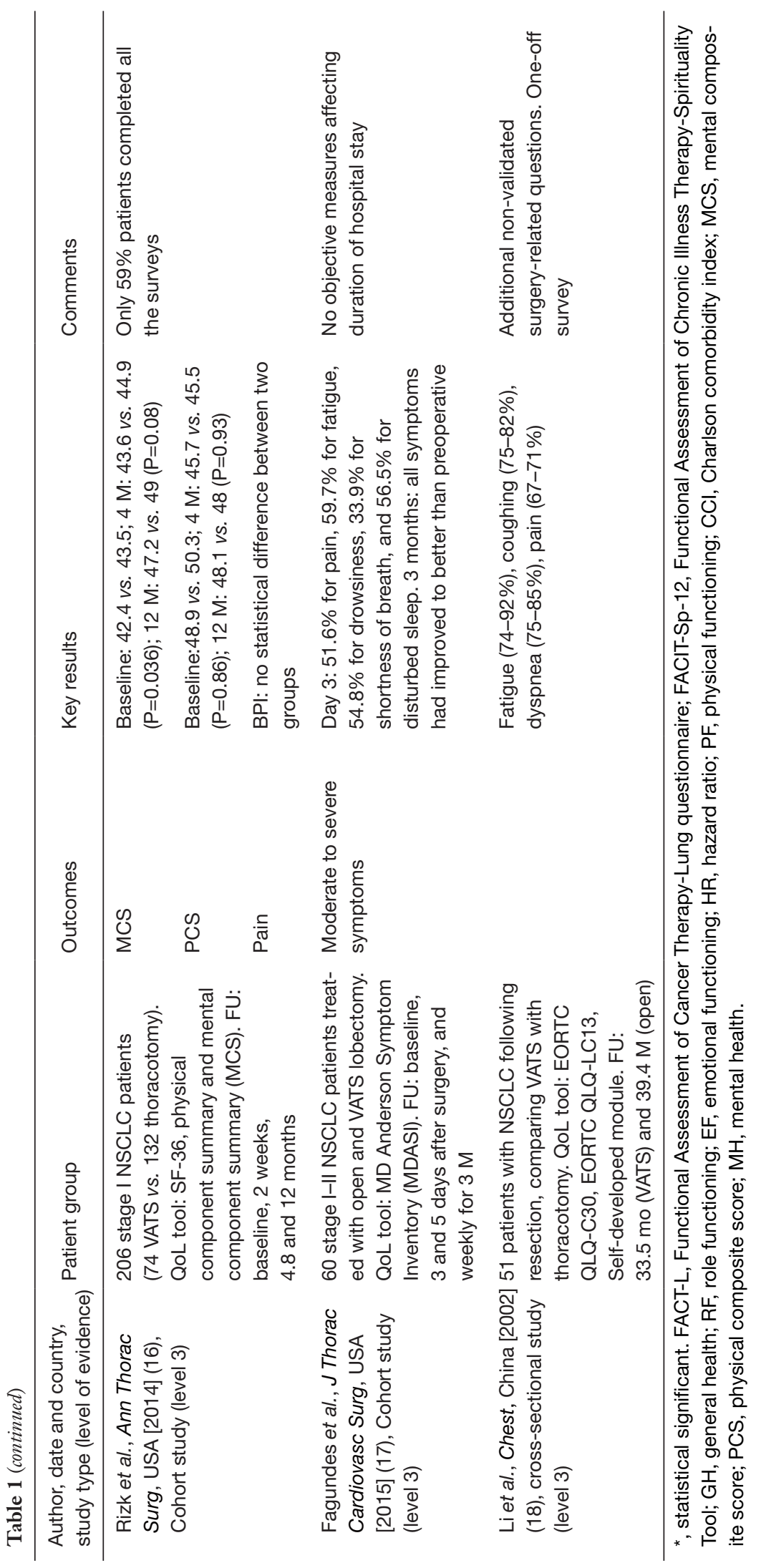


Widder et al. (7) looked prospectively at longitudinal changes of QoL parameters after SABR or threedimensional conformal radiotherapy (3D-CRT). They found that global QoL and physical functioning were stable at any follow-up within the first year. They also reported a statistically significant increase in dyspnea, although the observed changes were not clinically significant.

The Ferrero et al. (8) study of 30 patients is the only one to report a clinically and statistically significant increase in fatigue after 135 days.

Jain et al. (9) reported that dyspnea, fatigue and coughing to be worse at baseline in patients treated with SABR over 11 days compared to 4 days of treatment. However, more patients treated on 4 consecutive days experienced a clinically meaningful increase in dyspnea at 1 and 4 months after treatment.

Videtic et al. (10) conducted a small prospective study which did not find any statistical difference after 12 months in terms of QoL. They reported however, a 9-point drop from baseline to 12-week scores on the patients' UCSD dyspnea questionnaire, approaching clinical significance of 10 points.

Sun et al. (11) showed that QoL was not seriously impacted in a small cohort of 19 early-stage lung cancer patients after 12 months of follow-up. The functional domain had the lowest score of all the subscales measured with the Functional Assessment of Cancer Therapy-Lung (FACT-L).

\section{VATS studies}

The surgical studies investigating specifically the effect of minimally-invasive anatomical lung resection (studies with more than 20\% VATS) on QoL were characterized by small sample sizes and limited longitudinal assessments. Five out of 7 studies' primary aim is in fact the direct comparison between different surgical accesses (open versus thoracoscopic).

Bendixen et al. (12) conducted the first RCT describing the trajectory of pain and QoL of open versus VATS lobectomies for cancer. With a follow-up of 52 weeks, they found QoL in the VATS group was significantly better than that of an age-matched cohort from the Danish population. After two weeks the worst levels of QoL were observed and then QoL gradually improved over 52 weeks.

Burfied et al. (13) showed that QoL worsened at 3 months. However, at 6 and 12 months, all domains had returned to baseline except physical functioning, which remained below baseline in patients older than 70 years. EF improved postoperatively in older and younger patients.

Handy et al. (14) reported that compared with preoperative, 6-month postop VATS patients were not significantly different in physical function, role physical, role-emotional, social function, mental health or energy. Postoperative categories of bodily pain and general health were significantly improved over preoperative values in the VATS group.

Most recently Khullar et al. (15), in the first attempt to implement patient-reported-outcomes measures (PROMS) into national databases, evaluated 127 patients with the National Institutes of Health Patient-Reported Outcome Measurement Information System (PROMIS) platform. They confirmed a significant worsening in pain, fatigue, and sleep scores and a decrease in physical function after 1 month from the operation. By 6 months, these had generally improved toward baseline. Anxiety/fear and depression both significantly improved after the operation. In 2014, Rizk et al. (16) prospectively compared VATS and open lobectomies. In both groups, QoL scores improved throughout the 12 months, and pain scores approached baseline levels by 4 months.

Fagundes et al. (17), conducted an interesting investigation on weekly symptom assessments in surgical stage I patients from the third postoperative day to 3 months. All symptoms (except fatigue) returned to preoperative levels by the end of the first month. Fatigue remained the most persistent symptom during the study.

Li et al. (18) included surgery-related questions in their retrospective study and found that lung cancer patients following surgical treatment without recurrence had good QoL and high levels of functioning after a mean of 33.5 months follow-up, with no significant differences between the VATS and open groups.

\section{Clinical bottom line}

We acknowledge the paucity of evidence in PROMS evaluation for these treatment modalities. Only one small RCT ( $N=22$ patients) was identified that provided evidence addressing the specific question reporting that global health status deteriorates in more of the surgical patients compare to the SABR ones.

Sixteen studies provided supporting evidence but did not directly compared QoL between the two treatments. The overall impression from these studies which assessed a total 832 SABR patients and 686 receiving anatomical VATS 
resections, is that physical components of QoL decrease immediately after treatment up to 3 months, returning to baseline after 1 year. EF often supersedes the pre-operative values across treatments. Trials like the SABRTooth (20), STABLE-MATES (NCT01622621) (21) and VALOR (Veterans Affairs Lung-Cancer-Surgery or StereotacticRadiotherapy) (22) will give us information necessary to clarify this issue.

\section{Acknowledgements}

The authors appreciate Ms Emma Smyllie for helping in reviewing part of the papers and Ms Samantha Mason for methodological advice.

Funding: This work was supported by Yorkshire Cancer Research as part of the first author PhD (Grant: L399).

\section{Footnote}

Conflicts of Interest: The authors have no conflicts of interest to declare.

\section{References}

1. Aaronson NK, Ahmedzai S, Bergman B, et al. The European Organization for Research and Treatment of Cancer QLQ-C30: a quality-of-life instrument for use in international clinical trials in oncology. J Natl Cancer Inst 1993;85:365-76.

2. Louie AV, van Werkhoven E, Chen H, et al. Patient reported outcomes following stereotactic ablative radiotherapy or surgery for stage IA non-small-cell lung cancer: Results from the ROSEL multicenter randomized trial. Radiother Oncol 2015;117:44-8.

3. Lagerwaard FJ, Aaronson NK, Gundy CM, et al. PatientReported Quality of Life After Stereotactic Ablative Radiotherapy for Early-Stage Lung Cancer. J Thorac Oncol 2012;7:1148-54.

4. Mathieu D, Campeau MP, Bahig H, et al. Long-term quality of life in early-stage non-small cell lung cancer patients treated with robotic stereotactic ablative radiation therapy. Pract Radiat Oncol 2015;5:e365-73.

5. Ubels RJ, Mokhles S, Andrinopoulou ER, et al. Quality of life during 5 years after stereotactic radiotherapy in stage I non-small cell lung cancer. Radiat Oncol 2015;10:98.

6. van der Voort van Zyp NC, Prevost JB, van der Holt B, et al. Quality of life after stereotactic radiotherapy for stage I non-small-cell lung cancer. Int J Radiat Oncol Biol Phys
2010;77:31-7.

7. Widder J, Postmus D, Ubbels JF, et al. Survival and Quality of Life After Stereotactic or 3D-Conformal Radiotherapy for Inoperable Early-Stage Lung Cancer. Int J Radiat Oncol Biol Phys 2011;81:e291-e297.

8. Ferrero C, Badellino S, Filippi AR, et al. Pulmonary function and quality of life after VMAT-based stereotactic ablative radiotherapy for early stage inoperable NSCLC: a prospective study. Lung Cancer 2015;89:350-6.

9. Jain S, Poon I, Soliman H, et al. Lung stereotactic body radiation therapy (SBRT) delivered over 4 or 11days: A comparison of acute toxicity and quality of life. Radiother Oncol 2013;108:320-5.

10. Videtic GMM, Reddy CA, Sorenson L. A prospective study of quality of life including fatigue and pulmonary function after stereotactic body radiotherapy for medically inoperable early-stage lung cancer. Support Care Cancer 2013;21:211-8.

11. Sun V, Kim JY, Williams AC, et al. Quality of life and symptoms following stereotactic body radiotherapy in early-stage lung cancer patients. J Community Support Oncol 2014;12:407-14.

12. Bendixen M, Jørgensen OD, Kronborg C, et al. Postoperative pain and quality of life after lobectomy via video-assisted thoracoscopic surgery or anterolateral thoracotomy for early stage lung cancer: a randomised controlled trial. Lancet Oncol 2016;17:836-44.

13. Burfeind WR, Tong BC, O'Branski E, et al. Quality of life outcomes are equivalent after lobectomy in the elderly. J Thorac Cardiovasc Surg 2008;136:597-604.

14. Handy JR, Asaph JW, Douville EC, et al. Does videoassisted thoracoscopic lobectomy for lung cancer provide improved functional outcomes compared with open lobectomy? Eur J Cardiothorac Surg 2010;37:451-5.

15. Khullar OV, Rajaei MH, Force SD, et al. Pilot Study to Integrate Patient Reported Outcomes After Lung Cancer Operations Into The Society of Thoracic Surgeons Database. Ann Thorac Surg 2017;104:245-53.

16. Rizk NP, Ghanie A, Hsu M, et al. A prospective trial comparing pain and quality of life measures after anatomic lung resection using thoracoscopy or thoracotomy. Ann Thorac Surg 2014;98:1160-6.

17. Fagundes CP, Shi Q, Vaporciyan AA, et al. Symptom recovery after thoracic surgery: Measuring patientreported outcomes with the MD Anderson Symptom Inventory. J Thorac Cardiovasc Surg 2015;150:613-9.e2.

18. Li WW, Lee TW, Lam SS, et al. Quality of life following lung cancer resection: video-assisted thoracic surgery vs. 
thoracotomy. Chest 2002;122:584-9.

19. Osoba D, Rodrigues G, Myles J, et al. Interpreting the significance of changes in health-related quality-of-life scores. J Clin Oncol 1998;16:139-44.

20. Snee MP, McParland L, Collinson F, et al. The SABRTooth feasibility trial protocol: a study to determine the feasibility and acceptability of conducting a phase III randomised controlled trial comparing stereotactic ablative radiotherapy (SABR) with surgery in patients with peripheral stage I non-small cell lung cancer (NSCLC) considered to be at higher risk of complications from surgical resection. Pilot Feasibility Stud 2016;2:5.

21. Health UNIo. Stereotactic Body Radiotherapy (SBRT) Versus Sublobar Resection for High-Risk Patients NonSmall Lung Cancer 2015.

22. Affairs UDoV. CSP \#2005 - Veterans Affairs Lung Cancer Surgery Or Stereotactic Radiotherapy trial (VALOR 2015).
Cite this article as: Pompili C, Absolom K, Franks K, Velikova G. Are quality of life outcomes comparable following stereotactic radiotherapy and minimally invasive surgery for stage I lung cancer patients? J Thorac Dis 2018;10(12):70557063. doi: 10.21037/jtd.2018.11.89 